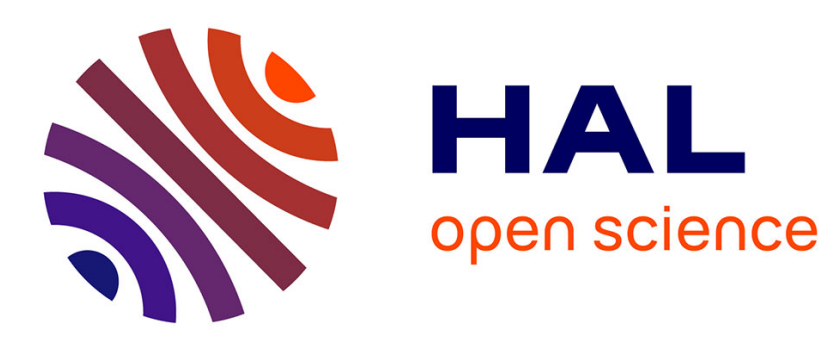

\title{
Qualité du pollen formé au froid chez la tomate (Lycopersicon esculentum Mill.). I. - Amélioration d'un test en conditions contrôlées
}

Brigitte Maisonneuve

\section{- To cite this version:}

Brigitte Maisonneuve. Qualité du pollen formé au froid chez la tomate (Lycopersicon esculentum Mill.). I. - Amélioration d'un test en conditions contrôlées. Agronomie, 1983, 3 (9), pp.873-878. hal-00884583

\section{HAL Id: hal-00884583 \\ https://hal.science/hal-00884583}

Submitted on 1 Jan 1983

HAL is a multi-disciplinary open access archive for the deposit and dissemination of scientific research documents, whether they are published or not. The documents may come from teaching and research institutions in France or abroad, or from public or private research centers.
L'archive ouverte pluridisciplinaire HAL, est destinée au dépôt et à la diffusion de documents scientifiques de niveau recherche, publiés ou non, émanant des établissements d'enseignement et de recherche français ou étrangers, des laboratoires publics ou privés. 


\title{
Qualité du pollen formé au froid chez la tomate (Lycopersicon esculentum Mill.). I. - Amélioration d'un test en conditions contrôlées
}

\author{
Brigitte MAISONNEUVE \\ I.N.R.A., Station d'Amélioration des Plantes maraîchères, Centre de Recherches agronomiques d'Avignon, \\ B.P. 94, F84140 Montfavet
} cours de différentes périodes de l'année afin d'améliorer un test établi précédemment : mesure de la qualité du pollen (p. 100 grains normaux) du $6^{e}$ au $17^{\circ}$ jour après la fin d'un traitement de 7 jours à $7{ }^{\circ} \mathrm{C}$. Nous confirmons, dans des expériences réalisées durant 6 mois, les résultats établis en octobre et février ; après une $1^{\text {ere }}$ chute assez brève de la qualité du pollen, une $2^{e}$ chute plus importante est observée pendant 2 à 6 jours entre le $7^{\mathrm{c}}$ et le $17^{\mathrm{c}}$ jour après la fin du traitement au froid ; plus les conditions en serre, avant et après le traitement, sont favorables à la floraison (forte luminosité, jours longs), plus la chute de qualité du pollen apparaît rapidement après le traitement.

La moyenne des mesures obtenues 5 jours consécutifs correspondant à la $2^{\mathrm{c}}$ chute de qualité du pollen $\left(7^{\mathrm{c}}\right.$ au $11^{\mathrm{c}}$ jour en mai ou septembre, $9^{\mathrm{e}}$ au $13^{\mathrm{c}}$ jour de fin mars à fin avril, $11^{\mathrm{e}}$ au $15^{\mathrm{c}}$ jour en octobre, $13^{\mathrm{c}}$ au $17^{\mathrm{e}}$ jour en février-mars) donne une bonne discrimination entre la variété sensible ("Coldset ») et les 2 variétés de moindre sensibilité («Précoce» et «Immuna Prior Beta»). Ainsi, il est possible, pendant 6 mois, de trier des variétés pour leur fertilité pollinique à basses températures en les comparant à 2 témoins : "Coldset " (très sensible) et "Précoce" (peu sensible).

Mots clés additionnels : Résistance au froid, méthode de test, chambre climatisée.

Quality of cold set pollen in tomato (Lycopersicon esculentum Mill.). I. - Improvement of a test under controlled conditions.

In a previous paper, a test was described to screen varieties for quality of pollen formed at low temperatures : plants were placed in a growth chamber at $7^{\circ} \mathrm{C}$ for 7 days; after this cold treatment, the plants were placed in a glasshouse to flower; from the $6^{\text {th }}$ until the $17^{\text {th }}$ day following the cold treatment, pollen quality at anthesis was measured by the percentage of normal grains. In 2 experiments, in October and February, the screening of varieties was good but the critical period was different in each month.

Therefore this test has been carried out for 6 months with 3 varieties. The first results found in a previous paper were confirmed : after a first short decrease of pollen quality, a second more important decrease was observed for 2 to 6 days between the $7^{\text {th }}$ and the $17^{\text {th }}$ day following the cold treatment. When the environment in the glasshouse before and after the cold treatment was relatively good (high light intensity, longer day), the pollen quality decrease occurred more rapidly after the cold treatment.

The mean of the measures on 5 consecutive days at the time of the second pollen quality decrease (between the $7^{\text {th }}$ and $11^{\text {th }}$ day in May or September, between the $9^{\text {th }}$ and $13^{\text {th }}$ day from end-March until end-April, between the $11^{\text {th }}$ and $15^{\text {th }}$ day in October, between the $13^{\text {th }}$ and $17^{\text {th }}$ day in February-March) gave good discrimination between the highly sensitive variety ("Coldset") and 2 less sensitive varieties ("Précoce " and "Immuna Prior Beta "). Therefore varieties could be screened for their pollen fertility at low temperature in 6 months, comparing them with the controls «Coldset» (high sensitivity) and «Précoce » (low sensitivity).

Additional key words : Cold resistance, testing method, growth chamber.

\section{INTRODUÇTION}

Dans un article précédent, nous avons rappelé le rôle prédominant du pollen dans la faible nouaison à basses températures chez la tomate, le pollen étant sensible pendant la microsporogenèse environ 2 semaines avant l'ouverture des fleurs (MAISONNEUvE, 1982). Nous avons montré qu'il était possible de classer des variétés pour leur aptitude à former du pollen fertile au froid d'après les résultats d'essais en conditions artificielles: les plantes 
cultivées en serre, sont transférées en chambre climatisée en début de floraison où elles subissent un traitement de $7 \mathrm{j}$ à $7^{\circ} \mathrm{C}$; puis la qualité du pollen est mesurée du $6^{\mathrm{e}}$ au $16^{\mathrm{e}}$ jour après le retour des plantes en serre; la moyenne des mesures obtenues durant $5 \mathrm{j}$ consécutifs a donné un bon classement variétal. Mais le choix de ces $5 \mathrm{j}$ était différent dans les 2 expériences réalisées, l'une en février, l'autre en octobre.

Les essais présentés ici ont donc pour but de définir la période de baisse de la qualité du pollen pour des traitements réalisés à différentes époques de l'année afin de choisir les $5 \mathrm{j}$ de mesures les plus discriminants pour des tests. Nous rappelons, dans cet article, les résultats de « Coldset », et « Précoce » dans les 2 expériences effectuées en octobre 1979 et en février 1980, déjà présentés dans l'article précédent, afin de faciliter la comparaison avec les résultats nouveaux présentés ici.

\section{MATERIEL ET MÉTHODES}

\section{A. Matériel végétal}

Nous étudions 3 variétés : "Coldset», «Précoce » et "Immuna Prior Beta ». La variété « Coldset» est choisie pour la forte sensibilité de son pollen au froid et « Précoce » pour sa moindre sensibilité d'après nos premières études en conditions contrôlées (MAISONNEUVE, 1982) ; à ces 2 variétés, est ajoutée la variété «Immuna Prior Beta» de moindre sensibilité (MAISONNEUVE, 1981).

\section{B. Méthodes}

\section{Conditions expérimentales}

Les 3 variétés sont étudiées au cours de 8 expériences réparties en hiver, printemps et automne, de 1979 à 1981 :

semis en décembre 1979 pour les expériences 1 et 2, en janvier 1980 pour l'expérience 3,

en février 1981 pour les expériences 4 et 5 ,

en mars 1981 pour l'expérience 6,

en juillet 1981 pour l'expérience 7 ,

en août 1979 pour l'expérience 8 .

La conduite des cultures est la même que celle cxposéc précédemment (MAISONNEUVE, 1982). Après 1 mois $1 / 2$ à 2 mois $1 / 2$ d'élevage en serre (température minimale de $14^{\circ} \mathrm{C}$ ), 4 plantes par variétés sont transférées en chambre climatisée pour un traitement de $7 \mathrm{j}$ de froid dans les 8 expériences. La température est basse nuit et jour, de $7 \pm 1{ }^{\circ} \mathrm{C}$ dans les expériences 1 à 7 . Elle est descendue à $2{ }^{\circ} \mathrm{C}$ la nuit tout au long de l'expérience 8 .

\section{Mesures}

La qualité du pollen est mesurée quotidiennement du $3^{\mathrm{e}}$, $5^{\mathrm{e}}$ ou $6^{\mathrm{e}}$ jour après la fin du traitement au froid jusqu'au $16^{\mathrm{e}}$ ou $17^{\mathrm{e}}$ jour. Nous prélevons le pollen d'une fleur à l'anthèse sur chacune des 4 plantes; 150 grains de pollen par fleur sont comptés dans une goutte de carmin acétique, soit 600 grains par date de mesure (parfois 450 ou 300 grains seulement quand il n'y a pas de fleurs épanouies sur certaines plantes). Le pourcentage de grains de pollen normaux sert d'estimation de la qualité du pollen (MAISONNEUVE, 1982). Les résultats sont présentés par date; le jour d'arrêt du traitement au froid sert d'origine des temps (jour 0 ).

\section{RESULTATS ET DISCUSSION}

Les qualités de pollen, mesurées au cours des 8 expériences, entre le $3^{\mathrm{e}}$ et le $17^{\mathrm{e}}$ jour après la fin du traitement de $7 \mathrm{j}$ de froid, sont présentées dans les tableaux 1,2 et 3 respectivement pour les 3 variétés "Coldset», "Précoce» et «Immuna Prior Beta ».

\section{A. Période d'action du traitement au froid}

Nous observons bien un effet du traitement au froid sur la qualité du pollen des fleurs qui s'ouvrent dans les 17 jours après la fin du traitement (tabl. $1,2 \& 3$ ). Cette baisse de qualité survient, en général, à 2 périodes séparées par quelques jours pendant lesquels la qualité du pollen produit est meilleure.

La $1^{\text {ere }}$ chute est, dans la plupart des cas, la moins intense et la plus courte ; elle dure 1 à $3 \mathrm{j}$ situés entre le $4^{\mathrm{e}}$ et le $10^{\mathrm{e}}$ jour après la fin du traitement; elle peut ne pas apparaître distincte de la $2^{c}$ chute ( "Coldset", expérience 8 ).

La $2^{\mathrm{e}}$ chute de qualité du pollen est régulièrement très marquée pendant 2 à $6 \mathrm{j}$ situés entre le $7^{\mathrm{e}}$ et le $17^{\mathrm{e}}$ jour après la fin du traitement. Elle est toujours plus intense que la $1^{\text {cre }}$ chute chez «Coldset»; mais, chez "Précoce" et «Immuna Prior Beta », elle peut être moins intense (expériences $1 \& 4$ ). Ces résultats confirment donc l'existence de 2 stades sensibles dans la microsporogenèse de la tomate montrés dans nos premiers essais (MAISONNEUVE, 1982). Le stade le plus sensible se situe à peu près dans la $2^{\mathrm{e}}$ semaine après la fin du traitement, ce qui correspond à la période sensible définie par ROBINSON et al. (1965). La $2^{\mathrm{c}}$ chute pourrait correspondre à une action du froid au moment de la méiose puisque RICK \& BOYNTON (1967) situent cette phase à peu près $10 \mathrm{j}$ avant l'anthèse. Nos observations concordent avec les études de IWAHORI (1965) sur l'effet des hautes températures sur la microsporogenèse chez la tomate; cet auteur trouve que la méiose, qui survient 9 à $8 \mathrm{j}$ avant l'anthèse, est sensible à un traitement à $40{ }^{\circ} \mathrm{C}$ en conditions artificielles. Dans cette étude, le stade sensible se prolonge jusqu'à $4 \mathrm{j}$ avant l'anthèse, époque de la $1^{\mathrm{cre}}$ mitose pollinique. Seules des études cytologiques dans nos conditions pourraient permettre de dire si la $1^{\text {ere }}$ chute de qualité du pollen, correspondant à un stade de la microsporogenèse postérieur à celui révélé par la $2^{\mathrm{e}}$ chute, résulte d'un traitement au froid pendant la mitose pollinique.

Comme le laissaient prévoir les 2 premières expériences réalisées (octobre 1979, expérience 8 ; février 1980, expérience 1), les dates où s'ouvrent les fleurs produisant un pollen de mauvaise qualité varient en fonction de l'époque de réalisation des expériences. Globalement, plus les jours sont longs et les rayonnements élevés dans la serre avant et après le traitement, plus la période de production de pollen de mauvaise qualité survient rapidement après la fin du traitement : par exemple, dans les expériences réalisées en février-début mars (expériences 1 et 2 ), la forte chute de qualité du pollen commence $13 \mathrm{j}$ après la fin du traitement, tandis que dans les expériences réalisées de fin mars à fin avril (expériences 3, 4 et 5) la forte chute commence le $10^{\mathrm{e}} \mathrm{j}$. La période où survient la $1^{\text {ère }}$ chute de qualité du pollen varie comme celle de la $2^{\mathrm{e}}$ chute ; mais en raison de la courte durée de cette $1^{\text {ère }}$ chute et du léger décalage entre variétés, il semble difficile de préciser les jours de son apparition en fonction des époques de traitement.

Ainsi, nous observons un très bon synchronisme variétal de la $2^{\mathrm{e}}$ chute de qualité du pollen. Ce synchronisme avait 
TABLEAU 1

Qualité du pollen de "Coldset" (p. 100 grains normaux) après un traitement de $7 j$ à basses températures.

«Coldset $»$ pollen quality (\% normal grains) after a 7-day cold treatment.

\begin{tabular}{|c|c|c|c|c|c|c|c|c|c|c|c|c|c|c|c|c|c|}
\hline \multirow{2}{*}{ Expérience } & \multirow{2}{*}{$\begin{array}{l}\text { Epoque du } \\
\text { traitement } \\
\text { au froid }\end{array}$} & \multicolumn{15}{|c|}{ Date : jour après la fin du traitement au froid } & \multirow{2}{*}{$\begin{array}{l}\text { Moyennes } \\
\text { de } 5 \text { jours } \\
\text { consécutifs* }\end{array}$} \\
\hline & & $3^{\mathrm{e}}$ & $4^{e}$ & $5^{\mathrm{c}}$ & $6^{e}$ & $7^{\mathfrak{c}}$ & $8^{e}$ & $9^{\mathrm{e}}$ & $10^{c}$ & $11^{\mathfrak{e}}$ & $12^{\mathrm{c}}$ & $13^{\mathrm{c}}$ & $14^{\mathrm{c}}$ & $15^{\mathrm{c}}$ & $16^{\mathrm{e}}$ & $17^{\mathrm{e}}$ & \\
\hline 1 & mi-février & - & - & 92 & 72 & 75 & 63 & 24 & 50 & - & 43 & $\underline{9}$ & $\underline{18}$ & $\underline{1}$ & $\underline{3}$ & $\underline{22}$ & 11 \\
\hline 2 & début mars & - & - & 86 & 74 & 86 & 10 & 2 & - & 9 & 43 & $\frac{\overline{9}}{9}$ & $\frac{5}{12}$ & $\frac{1}{1}$ & 0 & 二 & 6 \\
\hline 3 & fin mars & - & - & 70 & 22 & 37 & 62 & 47 & 0 & 1 & 17 & $\frac{1}{44}$ & $\frac{\frac{1}{9}}{91}$ & $9 \frac{1}{7}$ & $\frac{\bar{x}}{98}$ & - & 22 \\
\hline 4 & début avril & - & - & 81 & 42 & 5 & 37 & 63 & $2 \overline{3}$ & $\underline{0}$ & 0 & $\frac{1}{3}$ & 28 & 86 & 96 & 97 & 18 \\
\hline 5 & fin avril & - & - & 84 & 16 & 21 & 37 & $\underline{47}$ & $\frac{2 x}{7}$ & $\underline{0}$ & $\underline{0}$ & $\underline{1}$ & 31 & 75 & 72 & 97 & 11 \\
\hline 6 & fin mai & - & - & 54 & 65 & $\underline{59}$ & $\underline{62}$ & $\underline{4}$ & $\underline{\overline{1}}$ & $\underline{50}$ & $\overline{94}$ & 96 & 92 & 96 & 98 & - & 35 \\
\hline 7 & $\begin{array}{l}\text { début } \\
\text { septembre }\end{array}$ & 83 & 91 & 51 & 29 & $\underline{44}$ & $\underline{17}$ & $\underline{3}$ & $\underline{26}$ & $\underline{21}$ & 74 & 55 & 92 & 97 & 90 & - & 22 \\
\hline 8 & $\begin{array}{l}\text { début } \\
\text { octobre }\end{array}$ & - & - & - & 79 & 60 & 67 & 54 & 39 & $\underline{27}$ & $\underline{10}$ & $\underline{1}$ & $\underline{9}$ & $\underline{9}$ & 90 & 65 & 11 \\
\hline
\end{tabular}

*chiffres soulignés.

TABLEAU 2

Qualité du pollen de "Précoce" ( $p .100$ grains normaux) après un traitement de 7 j à basses températures.

"Précoce " pollen quality (\% normal grains) after a 7-day cold treatment.

\begin{tabular}{|c|c|c|c|c|c|c|c|c|c|c|c|c|c|c|c|c|c|}
\hline \multirow{2}{*}{ Expérience } & \multirow{2}{*}{$\begin{array}{l}\text { Epoque du } \\
\text { traitement } \\
\text { au froid }\end{array}$} & \multicolumn{15}{|c|}{ Date : jour après la fin du traitement au froid } & \multirow{2}{*}{$\begin{array}{l}\text { Moyennes } \\
\text { de } 5 \text { jours } \\
\text { consécutifs" }\end{array}$} \\
\hline & & $3^{\mathrm{c}}$ & $4^{\mathrm{c}}$ & $5^{\mathrm{e}}$ & $6^{\mathrm{e}}$ & $7^{\mathrm{c}}$ & $8^{\mathrm{e}}$ & $g^{e}$ & $10^{\mathrm{e}}$ & $11^{\mathrm{e}}$ & $12^{\mathrm{e}}$ & $13^{\mathrm{e}}$ & $14^{\mathrm{c}}$ & $15^{\mathrm{e}}$ & $16^{\mathrm{e}}$ & $17^{\mathrm{e}}$ & \\
\hline 1 & mi-février & - & - & - & 33 & 10 & 11 & 35 & 92 & 91 & 81 & 18 & 14 & 32 & $\underline{34}$ & 96 & 39 \\
\hline 2 & début mars & - & - & 74 & 48 & 91 & 33 & - & 67 & 83 & 84 & $\underline{34}$ & $\underline{10}$ & $\underline{27}$ & $\underline{36}$ & $\simeq$ & 27 \\
\hline 3 & fin mars & - & - & 87 & 58 & 63 & 96 & $\underline{94}$ & 43 & 15 & $\underline{86}$ & $\underline{\overline{96}}$ & $\frac{10}{93}$ & $\frac{21}{97}$ & $\frac{70}{97}$ & - & 67 \\
\hline 4 & début avril & - & - & 13 & 5 & 53 & 89 & $\overline{84}$ & $\overline{36}$ & $\overline{16}$ & $\overline{27}$ & $\overline{68}$ & - & 98 & 98 & 98 & 46 \\
\hline 5 & fin avril & - & - & 36 & 43 & 86 & 84 & $\frac{77}{77}$ & $\underline{25}$ & $\frac{2}{3}$ & 48 & $\frac{0 v}{61}$ & 96 & 85 & 97 & 96 & 43 \\
\hline 6 & fin mai & - & - & 43 & 88 & $\underline{82}$ & $\underline{56}$ & $\underline{35}$ & $\underline{16}$ & $\underline{7 \overline{7}}$ & $\overline{93}$ & $\frac{27}{97}$ & 95 & 97 & 97 & - & 53 \\
\hline 7 & $\begin{array}{l}\text { début } \\
\text { septembre }\end{array}$ & 79 & 16 & 28 & 70 & $\underline{23}$ & $\underline{5}$ & $\underline{31}$ & $\underline{22}$ & $\underline{91}$ & 97 & 93 & 93 & 97 & 97 & - & 34 \\
\hline 8 & $\begin{array}{l}\text { début } \\
\text { octobre }\end{array}$ & - & - & - & 33 & 19 & 54 & 86 & 84 & $\underline{49}$ & $\underline{15}$ & $\underline{26}$ & $\underline{23}$ & $\underline{69}$ & 99 & 96 & 36 \\
\hline
\end{tabular}

*chiffres soulignés.

TABLEAU 3

Qualité du pollen de "Immuna Prior Beta" (p. 100 grains normaux) après un traitement de 7 jà basses températures.

"Immuna Prior Beta" pollen quality (\% normal grains) after a 7-day cold treatment.

\begin{tabular}{|c|c|c|c|c|c|c|c|c|c|c|c|c|c|c|c|c|c|}
\hline Expérience & $\begin{array}{c}\text { Epoque du } \\
\text { traitement } \\
\text { au froid }\end{array}$ & $3^{e}$ & $4^{e}$ & $5^{\mathrm{e}}$ & Date & : jor & $\mathrm{r}$ apr & es la & fin $d$ & tra & temer & t $\mathrm{au}$ & froid & $15^{\mathrm{e}}$ & $16^{\mathrm{e}}$ & $17^{\mathrm{e}}$ & $\begin{array}{l}\text { Moyennes } \\
\text { des } 5 \text { jours } \\
\text { consécutifs* }\end{array}$ \\
\hline 1 & mi-février & - & - & - & 84 & 36 & 40 & 74 & 85 & 87 & 85 & 29 & 28 & 10 & $\underline{5}$ & 56 & 26 \\
\hline 2 & début mars & - & - & 72 & - & 64 & 72 & - & 17 & 85 & 86 & $\overline{62}$ & $\overline{0}$ & $\overline{42}$ & $\underline{10}$ & 二 & 29 \\
\hline 3 & fin mars & - & - & 80 & 50 & 71 & 93 & 96 & 36 & 15 & 61 & 99 & $\overline{93}$ & $\overline{92}$ & 99 & - & 61 \\
\hline 4 & début avril & - & - & 45 & 0 & 58 & 84 & $\overline{76}$ & $\overline{38}$ & $\overline{4}$ & $\overline{\underline{3}}$ & $\overline{31}$ & 97 & 98 & 98 & 98 & 30 \\
\hline 5 & fin avril & - & - & 49 & 88 & 92 & 93 & $\overline{92}$ & 60 & $\overline{0}$ & $3 \frac{\bar{x}}{8}$ & $\overline{77}$ & 74 & 92 & 98 & 98 & 53 \\
\hline 6 & fin mai & - & - & 77 & 93 & $\underline{90}$ & $\underline{60}$ & $\underline{1}$ & $\underline{1}$ & $\underline{69}$ & $\frac{57}{97}$ & $\overline{97}$ & 93 & 95 & 98 & - & 44 \\
\hline 7 & $\begin{array}{l}\text { début } \\
\text { septembre }\end{array}$ & 83 & 64 & 60 & 81 & $\underline{81}$ & $\underline{24}$ & $\underline{17}$ & $\underline{3}$ & $\underline{95}$ & 98 & 97 & 95 & 98 & 98 & - & 44 \\
\hline 8 & $\begin{array}{l}\text { début } \\
\text { octobre }\end{array}$ & - & - & - & 44 & 53 & 51 & 88 & 83 & $\underline{63}$ & $\underline{37}$ & $\underline{41}$ & $\underline{47}$ & $\underline{60}$ & 87 & 79 & 50 \\
\hline
\end{tabular}

*chiffres soulignés. 
déjà été observé dans les premières études (octobre 1979 et février 1980), non seulement pour « Coldset » et «Précoce ", mais aussi pour les 4 autres variétés qui étaient étudiées (MAISONNEUVE, 1982). Nous pouvons donc définir, pour chacune des 8 époques d'étude, la période de production de pollen de mauvaise qualité après $7 \mathrm{j}$ au froid : du $13^{\mathrm{c}}$ au $17^{\mathrm{e}} \mathrm{j}$ après un traitement entre la mi-février et début mars, du $11^{\mathrm{e}}$ au $15^{\mathrm{e}} \mathrm{j}$ début octobre, du $9^{\mathrm{e}}$ ou $10^{\mathrm{e}}$ au $13^{\mathrm{e}}$ ou $14^{\mathrm{e}} \mathrm{j}$ entre fin mars et fin avril, du $8^{\mathrm{e}}$ ou $9^{\mathrm{e}}$ au $10^{\mathrm{e}}$ ou $11^{\mathrm{e}} \mathrm{j}$ fin mai et du $7^{\mathrm{e}}$ ou $8^{\mathrm{e}}$ au $10^{\mathrm{e}}$ ou $11^{\mathrm{e}}$ jour début septembre.

\section{B. Sensibilité variétale}

1. Les minima, qui se situent pendant la $2^{\mathrm{e}}$ chute mais à des dates différentes selon les variétés dans une même expérience, ne diffèrent pas toujours entre «Coldset » et les variétés de moindre sensibilité (tabl. 1, 2 \& 3); par exemple, les minima sont 0 p. 100 dans l'expérience $2 \mathrm{chez}$ "Coldset» $\left(16^{\mathrm{e}} \mathrm{j}\right)$ comme chez «Immuna Prior Beta " $\left(14^{\mathrm{e}}\right.$ j) ainsi que dans l'expérience 5 pour ces 2 mêmes variétés $\left(11^{\mathrm{e}} \mathrm{j}\right)$; les minima ne diffèrent que très faiblement (3 p. 100 et 5 p. 100) dans l'expérience $7\left(9^{\mathrm{e}}, 8^{\mathrm{e}}\right.$ et $10^{\mathrm{e}} \mathrm{j}$ respectivement pour «Coldset », «Précoce » et «Immuna Prior Beta »). les minima ne permettent donc pas d'établir des différences variétales.

2. Les moyennes des mesures obtenues 11 j consécutifs, du $6^{\mathrm{C}}$ au $16^{\mathrm{C}} \mathrm{j}$ après la fin du traitement, montrent la plus grande sensibilité de «Coldset " par rapport à «Précoce » et à «Immuna Prior Beta » (fig. 1a). Les valeurs inférieures de ces moyennes chez «Coldset » proviennent d'une durée souvent plus longue et d'une intensité plus forte que celles de la $2^{\mathrm{e}}$ chute de qualité du pollen et, par ailleurs, de valeurs plus faibles chez «Coldset » des qualités de pollen mesurées entre les 2 chutes (tabl. 1, 2 \& 3). D'après l'analyse de variance de rang de Friedman, les différences variétales sont hautement significatives (seuil 0,001) ainsi que les différences entre expériences. Ces moyennes de $11 \mathrm{j}$ de mesures ne semblent pas toujours bien discriminer «Coldset» des 2 variétés à moindre sensibilité (traitement mi-février et fin mai, fig. 1a). De plus, le calcul de ces moyennes nécessite des observations de pollen pendant $11 \mathrm{j}$ consécutifs.

\section{Choix de 5 jours représentatifs de la sensibilité variétale}

Les résultats obtenus en mars, avril, mai et septembre confirmant ceux des premières expériences réalisées en février et octobre pour ce qui est des différences variétales, nous avons également calculé les moyennes des mesures obtenues $5 \mathrm{j}$ consécutifs. Nous choisissons, dans chaque expérience, les 5 j qui encadrent le mieux la $2^{c}$ chute de qualité du pollen pour les 3 variétés étudiées. Les moyennes ainsi calculées permettent de discriminer «Coldset " des 2 variétés de moindre sensibilité (fig. 1b). Une analyse de variance de rang de Friedman montre que les différences variétales sont hautement significatives (seuil $=0,001$ ) ainsi que les différences entre expériences. Ainsi "Coldset » est toujours la variété la plus sensible, «Précoce » est la moins sensible dans 4 expériences $(1,3,4,6)$ et «Immuna Prior Beta " est la moins sensible dans les 4 autres expériences. Les résultats obtenus en mars, avril, mai et septembre confirment donc ceux des premières expériences réalisées en février et octobre (MAISONNEUVE, 1982) : il est possible d'évaluer l'aptitude variétale à produire du pollen au froid par des mesures de pourcentages de grains normaux durant $5 \mathrm{j}$ après un traitement de $7 \mathrm{j}$ à $7{ }^{\circ} \mathrm{C}$.

Les $5 \mathrm{j}$ retenus sont mentionnés sur la figure $1 \mathrm{~b}$ :

- du $13^{\mathrm{e}}$ au $17^{\mathrm{e}}$ jour, pour les expériences effectuées en février-mars

- du $11^{\mathrm{e}}$ au $15^{\mathrm{e}}$ jour, pour les expériences effectuées en octobre

- du $9^{\mathrm{e}}$ au $13^{\mathrm{e}}$ jour, pour les expériences effectuées en mars-avril

- du $7^{\mathrm{e}}$ au $11^{\mathrm{e}}$ jour, pour les expériences effectuées en mai et en septembre.

En ce qui concerne les autres époques de l'année, certaines semblent défavorables à ce type de test; en particulier, les mois de novembre à janvier, au cours desquels des avortements de fleurs sont fréquents chez certaines variétés, peuvent être déconseillés. D'autre part, le niveau relativement élevé de la moyenne, calculée sur $5 \mathrm{j}$ consécutifs, fin mai chez «Coldset », n'incite pas à effectuer des tests en été ; en effet, cette forte valeur est due au petit nombre de jours de mesures présentant de basses valeurs de qualité du pollen (seulement 2 j avec moins de 50 p. 100 de

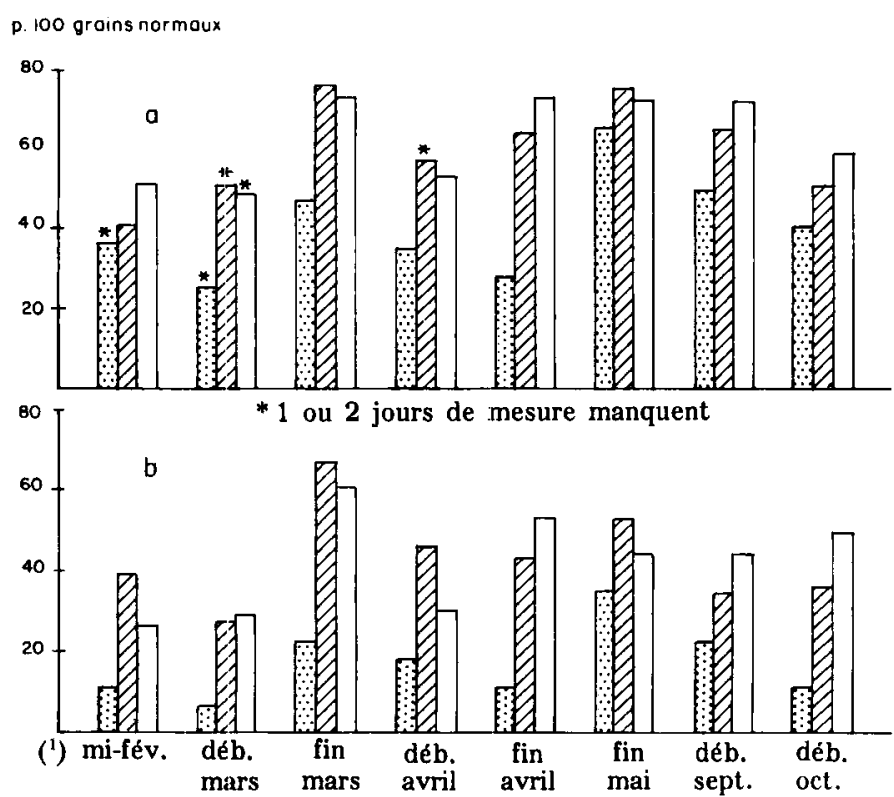

(2) $13^{\mathrm{e}} \rightarrow 17^{\mathrm{e}} \rightarrow 13^{\mathrm{e}} \rightarrow 16^{\mathrm{e}} \rightarrow 9^{\mathrm{e}} \rightarrow 13^{\mathrm{e}} \rightarrow 9^{\mathrm{e}} \rightarrow 13^{\mathrm{e}} \rightarrow 9^{\mathrm{e}} \rightarrow 13^{\mathrm{e}} \rightarrow 7^{\mathrm{e}} \rightarrow 11^{\mathrm{e}} \rightarrow 7^{\mathrm{e}} \rightarrow 11^{\mathrm{e}} \rightarrow 11^{\mathrm{e}} \rightarrow 15^{\mathrm{e}}$
Figure 1

Qualité du pollen (p. 100 grains normaux) de 3 variétés après un traitement de 7 jà basses températures.

a. Moyennes des mesures de 11 j consécutifs du $6^{e}$ au $16^{e}$ japrès la fin du traitement au froid.

$b$. Moyennes des mesures de 5 j consécutifs encadrant les minima de fertilité pollinique.

(1) Epoque du traitement au froid.

$\left({ }^{2}\right)$ Période de 5 jours considérée.

\section{$\therefore$ * Coldset " $\square \square$ " Précoce » $\square$ «Immuna Prior Beta 》}

Pollen quality (\% normal grains) in 3 varieties after a 7-day cold treatment

a. Means of results obtained in 11 days from the 6th until the 16th day after the end of treatments.

$b$. Means of results obtained in the 5 consecutive days which enclose the pollen fertility minima.

(1) Date of cold treatment.

(2) Five consecutive days period. 
grains normaux, tabl. 1) qui peut provenir d'une plus grande vitesse de développement des fleurs favorisée par les jours longs et le fort rayonnement en serre avant et après le traitement ; dans cette hypothèse, des tests réalisés en été donneraient une période de production de pollen de mauvaise qualité trop brève pour bien discriminer les variétés.

\section{CONCLUSION}

Ces expériences montrent qu'il est possible pendant 6 mois, de février à mai et en septembre-octobre, de trier des variétés pour leur aptitude à produire du pollen fertile au froid. En effet, après un traitement de $7 \mathrm{j}$ en conditions contrôlées à $7{ }^{\circ} \mathrm{C}$, des mesures des pourcentages de grains de pollen normaux chez des fleurs qui s'ouvrent $5 \mathrm{j}$ consécutifs bien choisis permettent d'obtenir un bon classement variétal; nous retrouvons la meilleure adaptation de «Précoce » par rapport à « Coldset » établie en serre froide (Maisonneuve \& Philouze, 1982). Ces 5 j de mesures, définis pour les 6 mois étudiés, "se situent entre le $7^{e}$ et le $17^{\mathrm{c}} \mathrm{j}$ après la fin du traitement.
Ce test, malgré la simplification apportée par la définition des 5 j de mesures qui évite les 11 ou 12 mesures journalières réalisées dans les premiers essais, semble encore assez lourd (élevage des plantes jusqu'à la floraison, transport des pots, plusieurs mesures). Quelques allègements au protocole ont pu déjà être effectués: semis direct en pots (3 graines semées, puis éclaircissage), pas de prémunition contre le virus de la mosaïque du tabac; de plus, il semblerait que la mesure de 2 fleurs au lieu de 4 fleurs par date donne un résultat identique.

Nous utilisons ce test à la Station de Montfavet pour la recherche de géniteurs de bonne fertilité au froid ; il est en effet impossible d'effectuer ce tri en conditions naturelles en raison des fluctuations climatiques journalières et annuelles. Les variétés à tester sont observées en même temps que «Coldset» et « Précoce » et classées par rapport à ces 2 témoins.

Reçu le 30 novembre 1982. Accepté le 9 mai 1983.

\section{RÉFÉRENCES BIBLIOGRAPHIQUES}

Iwahori S., 1965. High temperature injuries in tomato. IV. Development of normal flower buds and morphological abnormalities of flower buds treated with high temperature. J. Jap. Soc. hortic. Sci., 34 (1), 33-41.

Maisonneuve B., 1981. Recherche d'un test permettant de trier, en conditions contrôlées, des génotypes de tomate ayant une bonne fertilité au froid. Genetics and breeding of Tomato. Proc. Meeting Tomato Working Group, Eucarpia, Avignon (France), May 1981, 195-202.

Maisonneuve B., 1982. Effet d'un traitement à basses températures, en conditions contrôlées, sur la qualité du pollen de tomate (Lycopersicon esculentum Mill.). Agronomie, 2 (8), 755-764.
Maisonneuve B., Philouze J., 1982. Action des basses températures nocturnes sur une collection variétale de tomate. Il. Etude de la quantité et de la qualité du pollcn. Agronomie, 2 (5), 453-458.

Rick C. M., Boynton J. E., 1967. A temperature-sensitive malesterile mutant of the tomato. Am. J. Bot., 54 (5), 601-611.

Robinson R. W., Shannon S., Mishanec W., 1965. Low temperature influences pollen production and fruit set of tomatoes. Farm. Res., 31 (1), 13-15. 